\title{
724.
}

\section{ON THE DEFORMATION OF A MODEL OF A HYPERBOLOID.}

[From the Messenger of Mathematics, vol. viII. (1879), pp. 51, 52.]

THE following is a solution of Mr Greenhill's problem set in the Senate-House Examination, January 14, 1878.

"Prove that, if a model of a hyperboloid of one sheet be constructed of rods representing the generating lines, jointed at the points of crossing; then if the model be deformed it will assume the form of a confocal hyperboloid, and prove that the trajectory of a point on the model will be orthogonal to the system of confocal hyperboloids."

Let $\left(x_{1}, y_{1}, z_{1}\right),\left(x_{2}, y_{2}, z_{2}\right)$ be points on the generating line of

then

$$
\frac{x^{2}}{a^{2}}+\frac{y^{2}}{b^{2}}-\frac{z^{2}}{c^{2}}=1
$$

$$
\begin{aligned}
& \frac{x_{1}^{2}}{a^{2}}+\frac{y_{1}{ }^{2}}{b^{2}}-\frac{z_{1}{ }^{2}}{c^{2}}=1, \\
& \frac{x_{2}^{2}}{a^{2}}+\frac{y_{2}^{2}}{b^{2}}-\frac{z_{2}{ }^{2}}{c^{2}}=1, \\
& \frac{x_{1} x_{2}}{a^{2}}+\frac{y_{1} y_{2}}{b^{2}}-\frac{z_{1} z_{2}}{c^{2}}=1 ;
\end{aligned}
$$

or, what is the same thing, if

then

$$
\frac{x_{1}}{a}, \frac{y_{1}}{b}, \frac{z_{1}}{c}=p_{1}, q_{1}, r_{1} ; \frac{x_{2}}{a}, \frac{y_{2}}{b}, \frac{z_{2}}{c}=p_{2}, q_{2}, r_{2} ;
$$

$$
\begin{gathered}
p_{1}^{2}+q_{1}^{2}-r_{1}^{2}=1 \\
p_{2}^{2}+q_{2}^{2}-r_{2}^{2}=1 \\
p_{1} p_{2}+q_{1} q_{2}-r_{1} r_{2}=1
\end{gathered}
$$


Similarly, if $\left(\xi_{1}, \eta_{1}, \zeta_{1}\right),\left(\xi_{2}, \eta_{2}, \zeta_{2}\right)$ be points on generating line of

and if

$$
\frac{\xi^{2}}{\alpha^{2}}+\frac{\eta^{2}}{\beta^{2}}-\frac{\zeta^{2}}{\gamma^{2}}=1 \text {, }
$$

then

$$
\frac{\xi_{1}}{\alpha}, \frac{\eta_{1}}{\beta}, \frac{\zeta_{1}}{\gamma}=\mathrm{p}_{1}, \mathrm{q}_{1}, \mathrm{r}_{1} ; \frac{\xi_{2}}{\alpha}, \frac{\eta_{2}}{\beta}, \frac{\zeta_{2}}{\gamma}=\mathrm{p}_{2}, \mathrm{q}_{2}, \mathrm{r}_{2} ;
$$

$$
\begin{aligned}
& p_{1}{ }^{2}+q_{1}{ }^{2}-r_{1}{ }^{2}=1, \\
& p_{2}{ }^{2}+q_{2}{ }^{2}-r_{2}{ }^{2}=1, \\
& p_{1} p_{2}+q_{1} q_{2}-r_{1} r_{2}=1 .
\end{aligned}
$$
is, if

Hence if $\left(x_{1}, y_{1}, z_{1}\right),\left(\xi_{1}, \eta_{1}, \zeta_{1}\right)$ be corresponding points on the two surfaces, that

$$
\frac{x_{1}}{a}, \frac{y_{1}}{b}, \frac{z_{1}}{c}=\frac{\xi_{1}}{a}, \frac{\eta_{1}}{\beta}, \frac{\zeta_{1}}{\gamma},=p_{1}, q_{1}, r_{1},
$$

and similarly, if $\left(x_{2}, y_{2}, z_{2}\right),\left(\xi_{2}, \eta_{2}, \zeta_{2}\right)$ are corresponding points, that is, if

$$
\frac{x_{2}}{a}, \frac{y_{2}}{b}, \frac{z_{2}}{c}=\frac{\xi_{2}}{\alpha}, \frac{\eta_{2}}{\beta}, \frac{\zeta_{2}}{\gamma}=p_{2}, q_{2}, r_{2} ;
$$

then we have, as before, the system of three equations

$$
\begin{aligned}
& p_{1}{ }^{2}+q_{1}{ }^{2}-r_{1}{ }^{2}=1, \\
& p_{2}{ }^{2}+q_{2}{ }^{2}-r_{2}{ }^{2}=1, \\
& p_{1} p_{2}+q_{1} q_{2}-r_{1} r_{2}=1 .
\end{aligned}
$$

Then if the two surfaces are confocal, that is, if

we shall have

$$
\alpha^{2}, \beta^{2},-\gamma^{2}=a^{2}+h, b^{2}+h,-c^{2}+h,
$$

$$
\left(x_{1}-x_{2}\right)^{2}+\left(y_{1}-y_{2}\right)^{2}+\left(z_{1}-z_{2}\right)^{2}=\left(\xi_{1}-\xi_{2}\right)^{2}+\left(\eta_{1}-\eta_{2}\right)^{2}+\left(\zeta_{1}-\zeta_{2}\right)^{2} .
$$

For this equation is

$$
a^{2}\left(p_{1}-p_{2}\right)^{2}+b^{2}\left(q_{1}-q_{2}\right)^{2}+c^{2}\left(r_{1}-r_{2}\right)^{2}=\alpha^{2}\left(p_{1}-p_{2}\right)^{2}+\beta^{2}\left(q_{1}-q_{2}\right)^{2}+\gamma^{2}\left(r_{1}-r_{2}\right)^{2},
$$

that is,

$$
\left(p_{1}-p_{2}\right)^{2}+\left(q_{1}-q_{2}\right)^{2}-\left(r_{1}-r_{2}\right)^{2}=0,
$$

an equation which is obviously true in virtue of the above system of three equations.

Hence, if on confocal surfaces

$$
\frac{x^{2}}{a^{2}}+\frac{y^{2}}{b^{2}}-\frac{z^{2}}{c^{2}}=1, \quad \frac{\xi^{2}}{a^{2}+h}+\frac{\eta^{2}}{b^{2}+h}-\frac{\zeta^{2}}{c^{2}-h}=1,
$$

we take two points $P_{1}, P_{2}$ on the first, and $Q_{1}, Q_{2}$ the corresponding points on the second; then $P_{1}, P_{2}$ being on a generating line of the first surface, $Q_{1}, Q_{2}$ will be on a generating line of the second surface, and $P_{1} P_{2}$ will be $=Q_{1} Q_{2}$. The same is evidently true for the quadrilaterals $P_{1} P_{2} P_{3} P_{4}$ and $Q_{1} Q_{2} Q_{3} Q_{4}$, where $P_{1} P_{2}, P_{2} P_{3}$, $P_{3} P_{4}, P_{4} P_{1}$ are generating lines on the first surface: and therefore $Q_{1} Q_{2}, Q_{2} Q_{3}, Q_{3} Q_{4}$, $Q_{4} Q_{1}$ are generating lines on the second surface, which proves the theorem. 